\title{
The neglected elderly with diabetes - raising the standards in care homes
}

\author{
CHARLES FOX, ANNE KILVERT
}

\begin{abstract}
A significant number of elderly care home residents have diabetes and concerns have been raised about the quality of care in this setting. To address this, Diabetes UK produced updated guidelines for good clinical practice in 2010. A recent audit of the standard of diabetes care in residents of care homes in England has been carried out by the Institute of Diabetes for Older People (IDOP) in collaboration with the Association of British Clinical Diabetologists (ABCD). The audit captured data from 2,043 care homes (response rate $23 \%$ ), with 5,087 residents $(10.4 \%)$ reported to have diabetes in a total population of 48,978 . The main concerns raised were under-reporting of diabetes in this population, lack of policies for diabetes management, poor communication channels with primary care and lack of access to training for care home staff. There were specific clinical concerns about the management of hypoglycaemia and foot care. The audit makes a number of recommendations for raising standards. The three pillars on which high quality care must be based are mandatory training for all staff, effective communication with primary care and implementation of policies for good clinical care.

Br J Diabetes Vasc Dis 2014;14:109-111
\end{abstract}

Key words: audit, care homes, diabetes, elderly, frailty

\section{Introduction}

In the face of an exponential rise in the population of people with type 2 diabetes, it is understandable that health care planners have concentrated on overall provision of care for this very large cohort, without recognising the needs of individual subgroups. Recently, attention has focused on the 'lost tribes' of diabetes (young people with type 1 diabetes, prisoners, those with severe mental illness). However, an important and frequently overlooked group is residents with diabetes in care homes in whom the prevalence of diabetes is reported to be up to $27 \% .^{1}$

Northampton General Hospital, Cliftonville, Northampton NN1 5BD, UK

Address for correspondence: $\mathrm{Dr}$ Charles Fox

R\&D Unit, Northampton General Hospital, Cliftonville, Northampton

NN1 5BD, UK.

Tel: +44 (0)1604 545941

E-mail: charles@foxfam.demon.co.uk

http://dx.doi.org/10.15277/bjdvd.2014.031
Abbreviations and acronyms
$A B C D$
CQC
Association of British Clinical Diabetologists
IDOP
Care Quality Commission
Institute of Diabetes for Older People
YHPHO York and Humberside Public Health Observatory

Figure 1. Care Home Diabetes Audit ${ }^{2}$

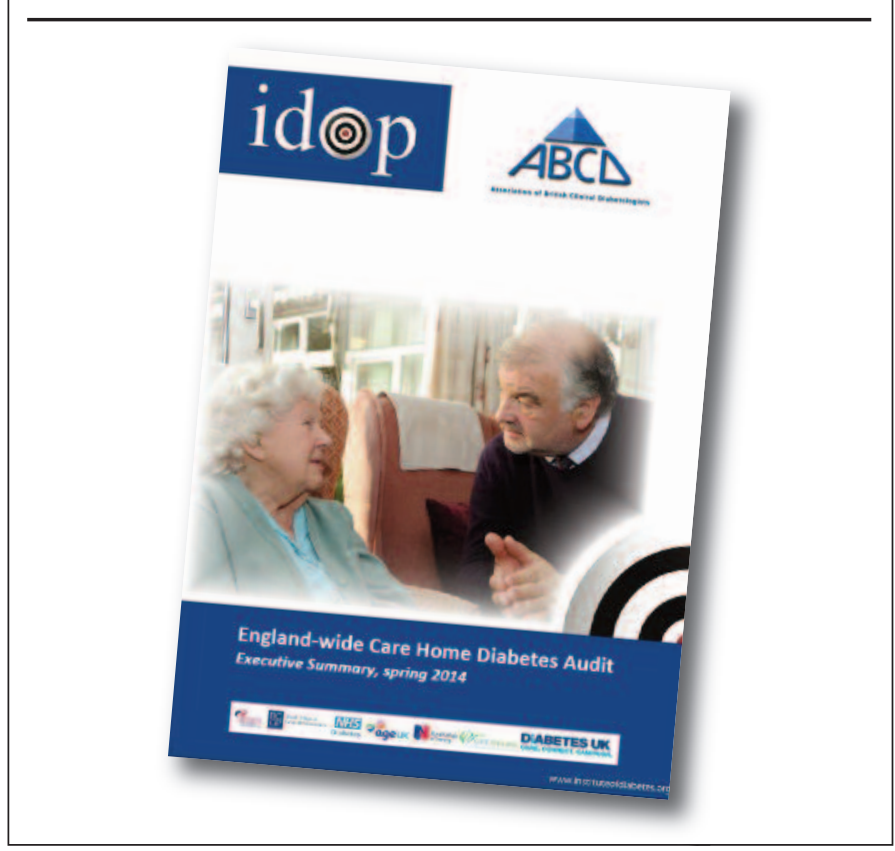

By definition, many care home residents are frail and/or have cognitive impairment. This complicates diabetes management and makes the resident more dependent on carers for day to day decisions relating to diabetes. For people who have taken pride in controlling their own diabetes, but no longer have the cognitive powers to achieve this, the loss of responsibility for their own care can be a source of frustration and distress and poor decision making by carers may lead to harm. This is a particular problem for people treated with insulin, but those on oral treatments may also come to harm if staff responsible for their care have an inadequate understanding of the needs of people with diabetes.

In the recent publication of a national audit of diabetes in care homes in England (Figure 1), ${ }^{2}$ IDOP in collaboration with $A B C D$ has identified areas of concern in the delivery of diabetes care to vulnerable elderly people in care homes, which is an important step forward in the campaign to raise standards of care in this sector. 
Table 1 Results of the audit of 2,043 care homes in England 2

1. Prevalence of diabetes

- $10.4 \%$ of residents were known to have diabetes $(5,087$ out of a total population of 48,978 )

2. Staff knowledge and education

- $47 \%$ of homes were unaware of Diabetes UK Care Home Guidelines

- $63 \%$ of homes had no nominated member of staff with responsibility for diabetes

- $33 \%$ of homes had no access to NHS training/education courses in diabetes for their staff

3. Policies for screening of residents for diabetes and other relevant conditions and for diabetes management

- $64 \%$ of homes had no policy for screening for diabetes

- $25 \%$ of residents had no documented test of cognition in the previous 12 months

- $32 \%$ of residents had no documented test of mood status in the previous 12 months

- $37 \%$ of care homes had no policy for management of hypoglycaemia

4. Individual resident assessment

- $47 \%$ of residents with diabetes were not assessed for knowledge of hypoglycaemia

- $17 \%$ of homes had no system to check whether self-medicating residents had taken their medication

- More than $53 \%$ of residents were at moderate or high risk of foot disease

5. Information sharing with primary care from the care home perspective

- $\quad 97 \%$ of residents with diabetes had an annual review with a GP but only $34 \%$ received a report from this review.

- $54 \%$ did not keep a record of the most recent $\mathrm{HbA}_{1 \mathrm{c}}$

- $58 \%$ did not keep a record of the latest kidney function test

\section{Existing guidance}

Diabetes UK recognised the importance of providing standards of care for residents in care homes in their 2010 document 'Good Clinical Practice Guidelines for Care Home Residents with Diabetes'. ${ }^{3}$ This comprehensive document aimed to set a standard for care of residents with diabetes and provided a template policy for diabetes care as well as a care plan.

\section{The England-wide Care Homes Audit}

The England-wide Care Homes Audit took place between September 2012 and late 2013, following a pilot in Bedfordshire and Hertfordshire.2 Questionnaires were sent to approximately 9,000 care homes in England and responses were received from $2,043(23 \%)$, of which 1,541 (75\% of respondents) had residents with diabetes.

\section{Audit findings and recommendations}

The main findings of the Care Homes Audit are shown in Table 1. The authors made a number of recommendations to improve the standards of care and these are summarised under the key areas listed in Table 2.
Table 2 Key areas identified to raise standards of diabetes care in care homes for the elderly ${ }^{2}$

- Clinical care, with particular focus on hypoglycaemia and feet

- Monitoring of health related aspects, with assessment of cognitive function, mood and continuing ability to self-medicate

- Communication with primary care to include documentation of annual review findings and relevant blood results

- Policies for management of diabetes to be implemented in line with the Diabetes UK Guidelines ${ }^{3}$

- Training and education to be provided for staff with a designated diabetes lead.

\section{Discussion}

Collecting accurate data is the first stage in any process which aspires to make improvements in a system of care. Despite a disappointing response rate of $23 \%$, this audit has identified important deficiencies in current practice. This has led to recommendations for improving standards of care and the onus is now on organisations such as Diabetes UK, ABCD and IDOP to work with the CQC to establish standards of care and ensure that these are met.

\section{Unanswered questions}

The questionnaire sent to the care homes is provided in an appendix. Understandably, the report itself does not detail the response to every question and the authors acknowledge the need to "drill deeper" into the data to extract more information. It would be interesting to learn the responses to the following questions:

- the prevalence of foot ulceration and access to podiatry

- the existence of a policy for diabetes management and individual care plans

- details of the extent and type of training staff had received.

\section{Prevalence of diabetes}

The first key finding in the audit report states that diabetes is 'highly prevalent' in care homes, but the figure (10.4\%) is considerably lower than the prevalence estimated by $\mathrm{YHPHO}$, which is $16.5 \%$ in the over-75 age group in England. ${ }^{4}$ One can speculate on the reasons for this and under-reporting is one possible explanation. Other prevalence studies of diabetes in care homes have been carried out in the last 15 years. A study of 30 care homes in the West Midlands found that $12.0 \%$ of residents had diagnosed diabetes and this rose to $26.7 \%$ after screening with an oral glucose tolerance test. ${ }^{1}$ A survey of 64 care homes in Lincolnshire recorded a $12.7 \%$ prevalence of known diabetes. ${ }^{5}$ Thus the prevalence of diabetes in this audit is only slightly lower than that quoted in these two UK studies.

\section{Poor communication and staff training}

The audit has highlighted poor communication between primary care and care homes; although nearly every resident had an annual diabetes review, only $34 \%$ of homes received a report of the results. This lack of communication echoes the main finding 


\section{Key messages}

To raise the quality of diabetes care:

- all care home staff should receive training in diabetes

- care homes should have policies for diabetes management

- communication between care providers is essential

of a focus group study, which investigated the views of care home workers with responsibility for residents with diabetes. ${ }^{6}$ The same focus groups also identified lack of staff training as a major concern and this has been confirmed by the present audit, in which a third of the care homes did not have access to diabetes education and training for their staff.

\section{What needs to be done?}

This England-wide audit provides the evidence to support anecdotal reports of inadequate care and poor standards for residents with diabetes. The next step is to build on this report by campaigning for the setting of national standards with a robust mechanism for ensuring that these are met. IDOP and $A B C D$ plan to work, together with the CQC, to establish standards of care and a system of accreditation, which must be met by all care homes. Mandatory training for all staff, effective communication with primary care and implementation of policies for good clinical care are the essential pillars on which a national system of high quality care must be built.

Conflict of interest CF has received a fee from IDOP for the role of Education Lead.

\section{Funding sources None.}

\section{References}

1. Sinclair AJ, Gadsby R, Penfold $\mathrm{S}$ et al. Prevalence of diabetes in care home residents. Diabetes Care 2001;24:1066-68. http://dx.doi.org/10.2337/diacare.24.6.1066

2. IDOP-ABCD. England-wide Care Home Diabetes Audit. Executive summary, spring 2014. June 2014. http://instituteofdiabetes.org/resources/ idop-publications and http://www.diabetologists-abcd.org.uk/Audits/ Care Home Diabetes Audit.pdf (accessed July 2014)

3. Diabetes UK. Good clinical practice guidelines for care home residents with diabetes. Diabetes UK, 2010

4. YHPHO. Key findings for England 2010. www.yhpho.org.uk (accessed July 2014)

5. Prestt A. Prevalence and diagnosis of diabetes in one PCT care home population. J Diabetes Nursing 2005;9:380.

6. Fox CJ, Kilvert A, Gillespie CR, Sinclair AJ. Diabetes care for the most vulnerable in society - the views of professionals working in care homes and domiciliary care using focus group methodology. Br J Diabetes Vasc Dis 2013;13:244-8. http://dx.doi.org/10.1177/1474651413506021

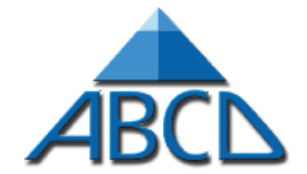

Association of British Clinical Diabetologists

\section{Dapagliflozin (Forxiga) Nationwide Audit Launching Soon!}

\title{
$A B C D$ is about to launch a nationwide audit of dapagliflozin in the UK.
}

This audit is particularly important with dapaglifozin being the first of a new class of drugs for diabetes, the SGLT2 inhibitors. We have a chance to assess real clinical efficacy and safety of this new type of treatment by pooling our experience nationwide

\section{Does your centre use dapagliflozin (Forxiga)?}

\author{
If yes, REGISTER YOUR CENTRE! by contacting abcd.audits@diabetologists.org.uk
}

- you will be able to analyse your local data easily

- you are invited to enter your patients' data into the online tool on N3 (the NHS secure network)

- the data will be automatically added to the national data in anonymised form

- we can provide easy-to-complete paper proformas for use in clinic if preferred

Please remember: - the more data, the more complete our understanding of this new treatment will be - all contributors will be listed in publications arising from data submission 